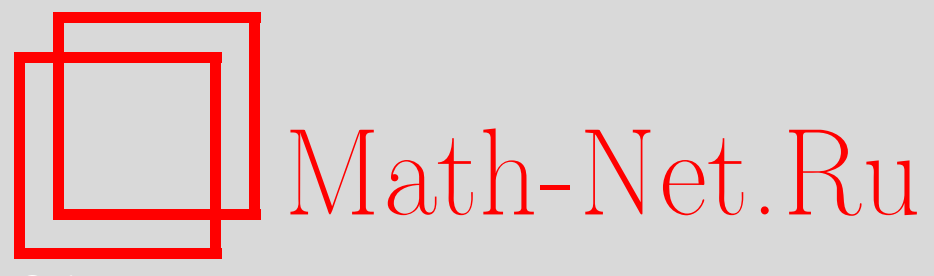

В. А. Попов, А. Л. Скубачевский, Гладкость обобщенных решений эллиптических дифференциально-разностных уравнений с вырождением вблизи границ подобластей, УМH, 2011, том 66, выпуск 6, 195-196

DOI: https://doi.org/10.4213/rm9437

Использование Общероссийского математического портала Math-Net.Ru подразумевает, что вы прочитали и согласны с пользовательским соглашением http://www . mathnet.ru/rus/agreement

Параметры загрузки:

IP : 52.23 .180 .231

26 апреля 2023 г., 12:35:24

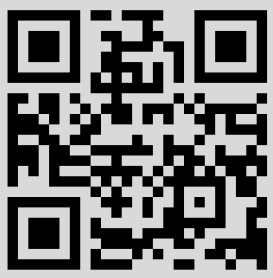




\title{
Гладкость обобщенных решений эллиптических дифференциально-разностных уравнений с вырождением вблизи границ подобластей
}

\author{
В. А. Попов, А. Л. Скубачевский
}

Эллиптические дифференциальные уравнения с вырождением изучались многими авторами (см. [1]-[3] и имеющуюся там библиографию). В работах [4], [5] рассматривались эллиптические дифференциально-разностные операторы $L_{R}$ порядка $2 m$ с вырождением вида $L_{R} u=L R u$, где $L$ - сильно эллиптический дифференциальный оператор, а $R$ - разностный оператор, эрмитова часть которого является неотрицательным вырожденным оператором. Интерес к таким операторам вызван приложениями полученных результатов к некоторым нелокальным эллиптическим задачам, возникающим в теории плазмы [6], а также появлением ряда принципиально новых свойств даже по сравнению с сильно эллиптическими дифференциально-разностными операторами.

Пусть $Q \subset \mathbb{R}^{n}$ - ограниченная область с границей $\partial Q \in C^{\infty}$. Пусть $\mathscr{M} \subset \mathbb{Z}^{n}-$ конечное множество. Обозначим через $M$ аддитивную абелеву группу, порожденную множеством $\mathscr{M}$. Обозначим через $Q_{r}$ открытые связные компоненты множества $Q \backslash$ $\bigcup_{h \in M}(\partial Q+h)$

ОПРЕДЕЛЕНИЕ 1 . Будем называть множества $Q_{r}$ подобластями, а совокупность $\mathscr{R}$ всевозможных подобластей $Q_{r}(r=1,2, \ldots)$ назовем разбиением области $Q$.

Легко видеть, что множество $\mathscr{R}$ не более чем счетно. Разбиение $\mathscr{R}$ естественным образом распадается на классы. Будем считать, что подобласти $Q_{r_{1}}, Q_{r_{2}} \in \mathscr{R}$ принадлежат одному и тому же классу, если существует вектор $h \in M$, для которого $Q_{r_{2}}=Q_{r_{1}}+h$. Обозначим подобласти $Q_{r}$ через $Q_{s l}$, где $s$ - номер класса $(s=1,2, \ldots)$, a $l$ - порядковый номер данной подобласти в $s$-м классе. Очевидно, что каждый класс состоит из конечного числа $N=N(s)$ подобластей $Q_{s l}$. Введем множество

$$
\mathscr{K}=\bigcup_{h_{1}, h_{2} \in M}\left\{\bar{Q} \cap\left(\partial Q+h_{1}\right) \cap \overline{\left[\left(\partial Q+h_{2}\right) \backslash\left(\partial Q+h_{1}\right)\right]}\right\} .
$$

Рассмотрим дифференциально-разностный оператор $L_{R}: D\left(L_{R}\right) \subset L_{2}(Q) \rightarrow L_{2}(Q)$, действующий по формуле

$$
L_{R} u=-\sum_{i, j=1}^{n} \frac{\partial^{2}}{\partial x_{i} \partial x_{j}} R_{i j} u \quad\left(u \in D\left(L_{R}\right)\right),
$$

с областью определения $D\left(L_{R}\right)=\dot{C}^{\infty}(Q)$, где $R_{i j} u=\sum_{h \in \mathscr{M}} a_{i j h} u(x+h)(i, j=1, \ldots, n)$, $\dot{C}^{\infty}(Q)$ - множество финитных бесконечно дифференцируемых функций в $Q$, $a_{i j h} \in \mathbb{C}$. Будем полагать $u(x)=0, x \in \mathbb{R}^{n} \backslash Q$.

Пусть $x \in \bar{Q}_{s 1}$ - произвольная точка. Рассмотрим все точки $x^{i} \in \bar{Q}$ такие, что $x^{i}-x \in M$. Поскольку область $Q$ ограниченная, множество $\left\{x^{i}\right\}$ состоит из конечного числа точек $I=I(s, x)(I \geqslant N(s))$. Занумеруем точки $x^{i}$ так, что $x^{i}=x+h_{s i}$ для $i=1, \ldots, N=N(s), x^{1}=x$, где $Q_{s i}=Q_{s 1}+h_{s i}$. Введем матрицы $R_{i j s}$ порядка $N \times N$

Работа выполнена при поддержке РФФИ (проекты № 10-01-00395, 09-01-00586), АВЦП (№ 2.1.1/5328) и ФЦП (№ 14.740.11.0691). 
и матрицы $\widetilde{R}_{i j s}(x)$ порядка $I \times I$ с элементами

$r_{k l}^{i j s}=\left\{\begin{array}{ll}a_{i j h}, & \text { если } h=h_{s l}-h_{s k} \in \mathscr{M}, \\ 0, & \text { если } h=h_{s l}-h_{s k} \notin \mathscr{M},\end{array} \quad \tilde{r}_{k l}^{i j s}(x)= \begin{cases}a_{i j h}, & \text { если } h=x^{l}-x^{k} \in \mathscr{M}, \\ 0, & \text { если } h=x^{l}-x^{k} \notin \mathscr{M} .\end{cases}\right.$

Обозначим через $\mathscr{N}(\cdot)$ и $\mathscr{R}(\cdot)$ соответственно ядро и образ некоторого оператора, $A_{i j s}=\left(R_{i j s}+R_{j i s}^{*}\right) / 2, B_{i j s}=\left(R_{i j s}-R_{j i s}^{*}\right) /(2 i), \widetilde{A}_{i j s}(x)=\left(\widetilde{R}_{i j s}(x)+\widetilde{R}_{j i s}^{*}(x)\right) / 2$, $\widetilde{B}_{i j s}(x)=\left(\widetilde{R}_{i j s}(x)-\widetilde{R}_{j i s}^{*}(x)\right) /(2 i)$.

Будем предполагать, что выполнены следующие условия.

УсловиЕ 1. Существуют самосопряженные неотрицательные разностные операторы $R_{i Q}$ такие, что справедливо неравенство

$$
\sum_{i, j=1}^{n} \widetilde{A}_{i j s}(x) \xi_{i} \xi_{j} \geqslant \sum_{i=1}^{n} \widetilde{R}_{i s}(x) \xi_{i}^{2} \geqslant 0
$$

для любых $\xi \in \mathbb{R}^{n}$ и для любых $x \in \bar{Q}_{s 1}$, где $\widetilde{R}_{i s}(x) \geqslant 0$ - матрицы, соответствующие операторам $R_{i Q}$.

УСловие 2. Множество $S=\left\{s: \operatorname{det} A_{i i s}=0, i=1, \ldots, n\right\}$ непусто.

УСловие 3. $\mathscr{N}\left(A_{i j s}\right) \subset \mathscr{N}\left(B_{i j s}\right), \mathscr{N}\left(A_{11 s}\right)=\mathscr{N}\left(A_{i i s}\right)=\mathscr{N}\left(R_{i s}\right), \mathscr{N}\left(A_{i i s}\right) \subset$ $\left(\mathscr{N}\left(A_{i j s}\right) \cap \mathscr{N}\left(A_{j i s}\right)\right), i, j=1, \ldots, n$, где $\mathscr{N}(\cdot)$ - ядро матрицы.

В работе [7] показано, что при выполнении условий 1-3 существует фридрихсово расширение $\mathscr{L}_{R}: D\left(\mathscr{L}_{R}\right) \subset L_{2}(Q) \rightarrow L_{2}(Q)$ оператора $L_{R}$. Известно, что решение уравнения $\mathscr{L}_{R} u=f$ может не принадлежать даже пространству Соболева $W_{2}^{1}(Q)$ при $f \in C^{\infty}(\bar{Q})$. Однако имеет место следующий результат о гладкости решений.

Теорема 1. Пусть выполнены условия 1-3. Пусть $и \in D\left(\mathscr{L}_{R}\right)$ является решением уравнения $\mathscr{L}_{R} u=f$, и пусть $f \in L_{2}(Q)$. Тогда $P^{R_{11}} u \in W_{2}^{2}\left(Q_{s l} \backslash \overline{\mathscr{K}}^{\varepsilon}\right)$ для любого $\varepsilon>0(s=1,2, \ldots ; l=1, \ldots, N(s))$, где $P^{R_{11}} u$-ортогональная проекция на образ разностного оператора $R_{11}, \mathscr{K}^{\varepsilon}=\{x \in \mathbb{R}: \operatorname{dist}(x, \mathscr{K})<\varepsilon\}$.

Теорема 1 обобщает результаты из [4], [5] о гладкости обобщенных решений эллиптических дифференциально-разностных уравнений с вырождением на случай, когда уравнение содержит несколько разностных операторов. С другой стороны, теория эллиптических дифференциально-разностных уравнений с вырождением позволяет исследовать достаточно широкий класс эллиптических задач с нелокальными условиями на компактных множествах, возникающих в теории плазмы [6].

\section{Список литературы}

[1] М. В. Келдыш, Докл. АН СССР, 77 (1951), 181-183. [2] Г. Фикера, Математика, 7:6 (1963), 99-121. [3] О. А. Олейник, Е. В. Радкевич, Итоги науки. Сер. Матем. Мат. анал. 1969, ВИНИТИ, М., 1971, 7-252. [4] А.Л. Скубачевский, Тр. ММО, 59, 1998, 240-285. [5] A.L. Skubachevskii, Elliptic functional differential equations and applications, Birkhäuser, Basel, 1997. [6] А. В. Бицадзе, А.А. Самарский, Докл. АН СССР, 185:4 (1969), 739-740. [7] В. А. Попов, А. Л. Скубачевский, Докл. РАН, 428:4 (2009), 450-453.

\section{В. А. Попов (V. A. Popov)}

Российский университет дружбы народов, Москва E-mail: volodimir.a@gmail.com

\section{А. Л. Скубачевский (А. L. Skubachevskii)}

Российский университет дружбы народов, Москва E-mail: skub@lector.ru
Представлено В. М. Бухштабером Принято редколлегией 13.07.2011 